\title{
Electronic excitations in a nonparabolic conduction band of an $n$-type narrow-gap semiconductor
}

\author{
Mitsutaka Yamaguchi, ${ }^{*}$ Takeshi Inaoka, ${ }^{\dagger}$ and Masayuki Hasegawa \\ Department of Materials Science and Technology, Faculty of Engineering, Iwate University, 4-3-5 Ueda, Morioka, Iwate 020-8551, Japan
}

(Received 29 May 2001; revised manuscript received 6 September 2001; published 8 February 2002)

\begin{abstract}
Taking full account of nonparabolicity of a conduction-band dispersion, we investigate those electronic excitations in the conduction band of an $n$-type narrow-gap semiconductor which are coupled with polar phonons. By incorporating the nonparabolic dispersion obtained by a $\mathbf{k} \cdot \mathbf{p}$ method in a complete manner into the random-phase approximation, we calculate the energy dispersion and the energy-loss intensity of two coupled plasmon-phonon modes and a longitudinal optical-phonon mode partially screened by carriers. The results are compared with those of two simplified treatments, namely, one with the spin-orbit splitting neglected and the other assuming a parabolic dispersion of the conduction band with its effective mass modified. This comparison asserts that complete treatment of the nonparabolic dispersion is indispensible to quantitative analysis of the plasmonlike mode at higher carrier concentrations. Simultaneously, it elucidates limitations and shortcomings of the simplified schemes. The complete treatment of the nonparabolicity leads to an excellent agreement with the experiment on the carrier-concentration dependence of an intensity-vanishing point in the infrared-reflection spectrum.
\end{abstract}

DOI: 10.1103/PhysRevB.65.085207

PACS number(s): 71.45.Gm, 77.22.-d, 78.30.-j

\section{INTRODUCTION}

Electronic excitations in a conduction band of $n$-type compound semiconductors, such as $n$-GaAs, $n$-InP, $n$-InAs, and $n-\mathrm{InSb}$, are in an energy regime of several tens to a hundred $\mathrm{meV}$, and mode energies of these excitations depend upon doping level. The above electronic excitations are coupled with polar optical phonons, when these two kinds of excitations lie in the same energy regime. The coupled plasmon (PL)-phonon (PH) modes can be observed by infraredreflection measurements and Raman-scattering measurements. ${ }^{1,2}$ The infrared-reflection spectrum is related to the angular-frequency $(\omega)$ dependence of the dielectric function at wave number $q=0$. In the Raman measurements, with change in incident laser frequency and scattering angle, we can observe the energy dispersion of the coupled PL-PH modes. In such an $n$-type polar semiconductor as mentioned above, combination of an extremely small effective mass and a large dielectric constant gives a large effective Bohr radius. Accordingly, increasing the doping level readily produces a carrier system of high effective density. In this case, the random-phase approximation (RPA) ${ }^{3}$ gives a quantitative description of the dynamical response of this carrier system. ${ }^{1,2,4-13}$ To examine coupling of electronic excitations and polar phonons, the RPA is combined with the Lorentzian-oscillator model that describes the phonon polarization. ${ }^{1,2,4-13}$ This combined scheme has been successfully applied to analysis of experimental data, especially the Raman measurements. The intensity of the energy loss involved in the dynamical response of our system is described by the energy-loss function. This function plays a central role in representing the Raman-scattering cross section [see, e.g., Eq. (75) in Ref. 1 or Eq. (2.96) in Ref. 2].

There exist three dispersion branches of coupled excitation modes [see, e.g., Figs. 6 and 18 in Ref. 1]. Two of these branches that lie on the smaller wave number $(q)$ side of the single-particle excitation (SPE) continuum originate from the PL-PH coupling. The coupled mode on each of these two branches decays away when it approaches or enters the SPE continuum. Another one of the three branches continues flat through the SPE continuum out to the larger- $q$ side of the same continuum. This branch can be identified as longitudinal optical-phonon modes that undergo a screening effect of carriers. We can examine the structure of coupled excitation modes on each branch by decomposing the induced charge density into a carrier component due to carrier density fluctuation and a phonon component arising from longitudinal phonon polarization. In Ref. 10, this decomposing analysis has shown how the mode structure varies with change in carrier concentration.

The conduction band of a compound semiconductor $\mathrm{GaAs}$, InP, InAs, or InSb is appreciably or highly nonparabolic, though almost isotropic. ${ }^{14,15}$ Especially, a narrow-gap semiconductor InAs or InSb has a remarkably nonparabolic conduction band. When this semiconductor is $n$ doped, the nonparabolicity exerts a significant influence on electronic excitations in the conduction band. There are several theoretical schemes to incorporate the effects of the nonparabolicity into the RPA. The simplest one of these schemes is to assume a parabolic dispersion with its effective mass modified. ${ }^{10,16}$ From now on, we call this scheme a modified parabolic (MP) one. In this scheme, the effective mass is so adjusted that the mode energies at wave number $q=0$ coincide with those obtained by accurate treatment of the nonparabolicity. This scheme gives a good description of excitation modes only in a small $q$ range. The second one is to represent the nonparabolic dispersion by a linear combination of $k^{2}, k^{4}$, and $k^{6}$ terms with their coefficients adjusted. ${ }^{9,13}$ Here the symbol $k$ is used to denote a wave number of each conduction-band state, while $q$ to stand for a wave number of each excitation mode. This scheme functions effectively for $\mathrm{GaAs}^{9}$ and $\mathrm{InP}^{13}$ with considerable band gaps where the nonparabolicity is not so significant. However, the above poly- 
nomial form cannot adequately describe such a highly nonparabolic dispersion as in InAs and InSb where an initial parabolic rise evolves into a linearlike increase with increase in $k$. A more advanced scheme is to employ a nonparabolic dispersion and a set of eigenfunctions obtained by a $\mathbf{k} \cdot \mathbf{p}$ method with the spin-orbit splitting neglected. ${ }^{12}$ Hereafter, we refer to this scheme as a simplified nonparabolic (SNP) one. This simplified scheme facilitates evaluation of the nonparabolic conduction-band dispersion, an overlap factor of eigenfunctions, and a numerical integral in the susceptibility of the carriers.

In the present work, we adopt a further improved scheme to incorporate into the RPA a nonparabolic dispersion and a set of eigenfunctions that are obtained in a complete manner by a k.p method including the spin-orbit splitting. We term this scheme a complete nonparabolic (CNP) one. As far as we know, this work is the first to attempt the CNP scheme. As an example for calculation, we take $n$-type InSb that has a remarkably nonparabolic conduction band. For several carrier concentrations, we calculate the dispersion relation and the energy-loss intensity of each of the three branches by means of the CNP, SNP, and MP schemes, and compare the results among the three schemes. This comparative analysis clarifies limitations and shortcomings of the two simplified schemes, the SNP and MP ones. In relation to the experiment, we calculate the carrier-concentration dependence of an intensity-vanishing point in the infrared-reflection spectrum. We compare the calculated results in the CNP and SNP schemes with the experimental results at various carrier concentrations. The present work is closely connected with the Raman-scattering measurements. However, there seem to be no Raman data for $n$-InAs or $n$-InSb with a highly nonparabolic conduction band. We anticipate such Raman data as can be compared with our calculations.

\section{THEORY}

In this section, we describe a theoretical framework for our following analysis. First, we briefly explain how oneelectron eigenstates and the dispersion relation of the conduction band can be obtained by virtue of Kane's theory. ${ }^{14}$ We pay attention to the cell-periodic factor $u_{\mathbf{k} \lambda}(\mathbf{r})$ of each Bloch function $\phi_{\mathbf{k} \lambda}(\mathbf{r})\left[=\exp (i \mathbf{k} \cdot \mathbf{r}) u_{\mathbf{k} \lambda}(\mathbf{r})\right]$ with wave vector $\mathbf{k}$ and spin orientation $\lambda$. The Schrödinger equation for $u_{\mathbf{k} \lambda}(\mathbf{r})$ is expressed as

$$
\left[\frac{\mathbf{p}^{2}}{2 m_{0}}+V(\mathbf{r})+\frac{\hbar}{m_{0}} \mathbf{k} \cdot \mathbf{p}+H_{\mathrm{SO}}\right] u_{\mathbf{k} \lambda}(\mathbf{r})=E^{\prime}(\mathbf{k}) u_{\mathbf{k} \lambda}(\mathbf{r}),
$$

with $E^{\prime}(\mathbf{k})=E(\mathbf{k})-\hbar^{2} \mathbf{k}^{2} / 2 m_{0}$. The symbols $m_{0}, \mathbf{p}, V(\mathbf{r})$, $H_{\mathrm{SO}}$, and $E(\mathbf{k})$ denote the free-electron mass, the momentum operator, a periodic potential, the spin-orbit coupling, and the energy dispersion of each band, respectively. As a basis set for representing $u_{\mathbf{k} \lambda}(\mathbf{r})$, we take an $s$-symmetry function and three $p$-symmetry ones with both spin orientations for each function. These functions are given as solutions of Eq. (1) for $k=0$ in the absence of $H_{\text {SO }}$. Then, Eq. (1) can be reduced to an eigenvalue problem of an $8 \times 8$ matrix, which results in one dispersion branch of the conduc- tion band and three branches of the valence band. Because of two possible spin orientations, each energy level on one of these four branches involves two degenerate eigenstates, which are labeled by $\lambda=1$ and 2 . The energy $E(\mathbf{k})$ of the conduction band can be obtained by taking the largest solution of an algebraic equation for $E^{\prime}\left[=E(\mathbf{k})-\hbar^{2} \mathbf{k}^{2} / 2 m_{0}\right]$ :

$E^{\prime}\left(E^{\prime}+E_{G}\right)\left(E^{\prime}+E_{G}+\Delta_{\mathrm{SO}}\right)-P^{2} \mathbf{k}^{2}\left(E^{\prime}+E_{G}+2 \Delta_{\mathrm{SO}} / 3\right)=0$,

where $E_{G}$ and $\Delta_{\text {SO }}$ signify the band-gap energy and the spinorbit splitting, respectively. The coefficient $P$ can be determined by

$$
P^{2}=\frac{3}{2} \hbar^{2}\left(\frac{1}{m_{0}^{*}}-\frac{1}{m_{0}}\right) /\left(\frac{2}{E_{G}}+\frac{1}{E_{G}+\Delta_{\mathrm{SO}}}\right),
$$

with the band-edge effective mass $m_{0}^{*}$. If we take a $z$ axis in the direction of $\mathbf{k}$, the cell-periodic functions $u_{\mathbf{k} \lambda}(\mathbf{r})$ in the conduction band can be written as

$$
u_{\mathbf{k} 1}(\mathbf{r})=a_{\mathbf{k}}|i S \downarrow\rangle+b_{\mathbf{k}}|(X-i Y) \uparrow\rangle / \sqrt{2}+c_{\mathbf{k}}|Z \downarrow\rangle,
$$

and

$$
u_{\mathbf{k} 2}(\mathbf{r})=a_{\mathbf{k}}|i S \uparrow\rangle+b_{\mathbf{k}}|-(X+i Y) \downarrow\rangle / \sqrt{2}+c_{\mathbf{k}}|Z \uparrow\rangle,
$$

in terms of three real coefficients

$$
\begin{gathered}
a_{\mathbf{k}}=P k\left(E^{\prime}+E_{G}+2 \Delta_{\mathrm{SO}} / 3\right) / N, \\
b_{\mathbf{k}}=\left(\sqrt{2} \Delta_{\mathrm{SO}} / 3\right) E^{\prime} / N,
\end{gathered}
$$

and

$$
c_{\mathbf{k}}=E^{\prime}\left(E^{\prime}+E_{G}+2 \Delta_{\mathrm{SO}} / 3\right) / N .
$$

In Eqs. (4) and (5), capitals $S, X, Y$, and $Z$ designate the symmetry of the basis functions, and arrows indicate the spin orientation. When the wave vector $\mathbf{k}$ is not in the $z$ direction, each basis function in a ket must be rotated in such a way that the new $z$ axis is oriented toward the $\mathbf{k}$ direction. The solution $E^{\prime}$ of Eq. (2) corresponding to the conduction band is substituted into Eqs. (6)-(8). The quantity $N$ is a normalizing factor defined by the square root of the sum of the squares of the numerators.

Based upon the conduction-band states obtained above, we calculate those electronic excitations in the nonparabolic conduction band which are coupled with polar phonons. In our $n$-type polar semiconductors with large effective Bohr radii, increasing the doping level readily creates such a high effective density of carriers that an impurity band due to donors merges into the conduction band. In this case, ionized donors can be smeared out into a uniform distribution of positive charges. Our system is composed of the carriers in the conduction band and a polarizable polar background. As mentioned in Sec. I, we employ the RPA combined with the Lorentzian-oscillator model. We consider the dynamical response of our system to a periodic and oscillatory external potential with wave vector $\mathbf{q}$ and angular frequency $\omega$. This response can be described by the following dielectric function: 


$$
\varepsilon(\mathbf{q}, \omega)=\varepsilon_{\mathrm{PH}}(\omega)-\left(4 \pi e^{2} / \mathbf{q}^{2}\right) \chi(\mathbf{q}, \omega) .
$$

The dielectric function $\varepsilon_{\mathrm{PH}}(\omega)$ to describe the phonon and background polarization can be expressed as

$$
\varepsilon_{\mathrm{PH}}(\omega)=\varepsilon_{\infty}+\frac{\left(\varepsilon_{0}-\varepsilon_{\infty}\right) \omega_{\mathrm{TO}}^{2}}{\omega_{\mathrm{TO}}^{2}-\omega^{2}-i \gamma \omega},
$$

where $\omega_{\text {TO }}$ is the transverse optical-phonon frequency, $\varepsilon_{\infty}$ and $\varepsilon_{0}$ are the high-frequency and static dielectric constants in the absence of carriers, respectively, and $\gamma$ is the phonon relaxation-rate constant. This local response scheme for optical phonons is a good approximation for our excitation modes whose wavelength is much longer than lattice constants. The susceptibility $\chi(\mathbf{q}, \omega)$ to describe the response of the carriers can be written in terms of Bloch functions $\phi_{\mathbf{k} \lambda}(\mathbf{r})$ as follows:

$$
\begin{aligned}
\chi(\mathbf{q}, \omega)= & \frac{1}{V_{0}} \sum_{\mathbf{k}, \lambda} \sum_{\mathbf{k}^{\prime}, \lambda} \frac{f\left(\mathbf{k}^{\prime}\right)-f(\mathbf{k})}{E\left(\mathbf{k}^{\prime}\right)-E(\mathbf{k})+\hbar \omega+i \hbar \eta} \\
& \times \int d^{3} \mathbf{r} \int d^{3} \mathbf{r}^{\prime} \exp (-i \mathbf{q} \cdot \mathbf{r}) \exp \left(i \mathbf{q} \cdot \mathbf{r}^{\prime}\right) \\
& \times \phi_{\mathbf{k} \lambda}^{*}\left(\mathbf{r}^{\prime}\right) \phi_{\mathbf{k}^{\prime} \lambda^{\prime}}\left(\mathbf{r}^{\prime}\right) \phi_{\mathbf{k}^{\prime} \lambda^{\prime}}^{*}(\mathbf{r}) \phi_{\mathbf{k} \lambda}(\mathbf{r}) \\
= & \frac{1}{V_{0}} \sum_{\mathbf{k}, \lambda} \sum_{\mathbf{k}^{\prime}, \lambda} \frac{f\left(\mathbf{k}^{\prime}\right)-f(\mathbf{k})}{E\left(\mathbf{k}^{\prime}\right)-E(\mathbf{k})+\hbar \omega+i \hbar \eta} \\
& \times\left|\int d^{3} \mathbf{r} \exp (-i \mathbf{q} \cdot \mathbf{r}) \phi_{\mathbf{k}^{\prime} \lambda^{\prime}}^{*}(\mathbf{r}) \phi_{\mathbf{k} \lambda}(\mathbf{r})\right|^{2}
\end{aligned}
$$

where $f(\mathbf{k}), V_{0}$, and $\eta$ denote the Fermi-Dirac distribution function, the system volume, and a small positive constant, respectively. Substituting $\phi_{\mathbf{k} \lambda}(\mathbf{r})=\exp (i \mathbf{k} \cdot \mathbf{r}) u_{\mathbf{k} \lambda}(\mathbf{r})$ in Eq. (11), using the periodicity of $u_{\mathbf{k} \lambda}(\mathbf{r})$, and converting the $\mathbf{k}$ sum into a $\mathbf{k}$ integral, we obtain ${ }^{12,17}$

$$
\begin{aligned}
\chi(\mathbf{q}, \omega)= & 2 \int \frac{d^{3} \mathbf{k}}{(2 \pi)^{3}} f(\mathbf{k})\left[\frac{I_{\mathbf{k}, \mathbf{k}+\mathbf{q}}}{E(\mathbf{k})-E(\mathbf{k}+\mathbf{q})+\hbar \omega+i \hbar \eta}\right. \\
& \left.+\frac{I_{\mathbf{k}, \mathbf{k}-\mathbf{q}}}{E(\mathbf{k})-E(\mathbf{k}-\mathbf{q})-\hbar \omega-i \hbar \eta}\right]
\end{aligned}
$$

where the overlap integral $I_{\mathbf{k}, \mathbf{k}^{\prime}}$ is defined by

$$
I_{\mathbf{k}, \mathbf{k}^{\prime}}=\frac{1}{2} \sum_{\lambda, \lambda^{\prime}}\left|\int u_{\mathbf{k}^{\prime} \lambda^{\prime}}^{*}(\mathbf{r}) u_{\mathbf{k} \lambda}(\mathbf{r}) d^{3} \mathbf{r}\right|^{2} .
$$

After a straightforward calculation with the aid of Eqs. (4) and (5), the integral $I_{\mathbf{k}, \mathbf{k}^{\prime}}$ can be expressed as ${ }^{18}$

$$
\begin{aligned}
I_{\mathbf{k}, \mathbf{k}^{\prime}}= & \left\{a_{\mathbf{k}} a_{\mathbf{k}^{\prime}}+\left(b_{\mathbf{k}} b_{\mathbf{k}^{\prime}}+c_{\mathbf{k}} c_{\mathbf{k}^{\prime}}\right) \cos \theta_{\mathbf{k}, \mathbf{k}^{\prime}}\right\}^{2} \\
& +(1 / 2)\left\{b_{\mathbf{k}} b_{\mathbf{k}^{\prime}} / \sqrt{2}-\left(b_{\mathbf{k}} c_{\mathbf{k}^{\prime}}+c_{\mathbf{k}} b_{\mathbf{k}^{\prime}}\right)\right\}^{2} \sin ^{2} \theta_{\mathbf{k}, \mathbf{k}^{\prime}},
\end{aligned}
$$

where $\theta_{\mathbf{k}, \mathbf{k}^{\prime}}$ designates the angle between $\mathbf{k}$ and $\mathbf{k}^{\prime}$. The energy $E(\mathbf{k})$ in Eq. (12) and the coefficients $a_{\mathbf{k}}, b_{\mathbf{k}}$, and $c_{\mathbf{k}}$ in Eq. (14) can be obtained from Eq. (2) and Eqs. (6)-(8), respectively. The chemical potential (Fermi level) $\mu$ for carrier concentration $n_{0}$ given can be determined by the relation

$$
2 \int \frac{d^{3} \mathbf{k}}{(2 \pi)^{3}} f(\mathbf{k})=n_{0},
$$

with $E(\mathbf{k})$ incorporated in $f(\mathbf{k})$. We rewrite the $\mathbf{k}$ integral in Eq. (12) in terms of spherical polar coordinates, and perform a numerical calculation of the resulting integral.

Next, we consider the energy loss involved in the response of our system to the external potential $U(\mathbf{q}, \omega)$. The energy loss per unit volume and per unit time can be expressed as

$$
W=\frac{\mathbf{q}^{2} \omega}{2 \pi}|U(\mathbf{q}, \omega)|^{2} \operatorname{Im}\left[-\frac{1}{\varepsilon(\mathbf{q}, \omega)}\right],
$$

where Im stands for the imaginary part. In view of this expression, we define the energy-loss function $F(\mathbf{q}, \omega)$ by

$$
F(\mathbf{q}, \omega)=\operatorname{Im}[-1 / \varepsilon(\mathbf{q}, \omega)] .
$$

Here, we pay attention to the $\omega$ dependence of $\varepsilon(\mathbf{q}, \omega)$ at $q$ $=0$. We take the limit of $q \rightarrow 0$ and ignore the relaxation-rate constants $\gamma$ and $\eta$. In this condition, the susceptibility $\chi(\mathbf{q}, \omega)$ can be expanded in powers of $1 / \omega$, and its expression up to the second order is given by

$$
\begin{aligned}
\chi(\mathbf{q}, \omega) \approx & \frac{2}{\hbar \omega} \int \frac{d^{3} \mathbf{k}}{(2 \pi)^{3}} f(k)\left(I_{\mathbf{k}, \mathbf{k}+\mathbf{q}}-I_{\mathbf{k}, \mathbf{k}-\mathbf{q}}\right) \\
& +\frac{2}{(\hbar \omega)^{2}} \int \frac{d^{3} \mathbf{k}}{(2 \pi)^{3}} f(k)\left[I_{\mathbf{k}, \mathbf{k}+\mathbf{q}}\{E(|\mathbf{k}+\mathbf{q}|)-E(k)\}\right. \\
& \left.+I_{\mathbf{k}, \mathbf{k}-\mathbf{q}}\{E(|\mathbf{k}-\mathbf{q}|)-E(k)\}\right]
\end{aligned}
$$

Here, the function $f(\mathbf{k})$ and the energy $E(\mathbf{k})$ are expressed as $f(k)$ and $E(k)(k=|\mathbf{k}|)$, respectively, to emphasize that these quantities depend not on the direction of $\mathbf{k}$ but only on the magnitude of $\mathbf{k}$. In view of the denominator $\mathbf{q}^{2}$ in Eq. (9), we calculate $\chi(\mathbf{q}, \omega)$ up to the second order in $\mathbf{q}$. For simplicity of calculation, we take the $z$ axis in the direction of q. The overlap integral $I_{\mathbf{k}, \mathbf{k} \pm \mathbf{q}}$ and the energy spacing $E(|\mathbf{k} \pm \mathbf{q}|)-E(k)$ can be expanded up to the second order in $q_{z}$ as follows:

$$
\begin{aligned}
I_{\mathbf{k}, \mathbf{k} \pm \mathbf{q}} \approx 1 & -\left[G(k)\left(\frac{k_{z}}{k}\right)^{2}+H(k) \frac{k^{2}-k_{z}^{2}}{k^{4}}\right] q_{z}^{2} \\
E(|\mathbf{k} \pm \mathbf{q}|)-E(k) \approx & \pm \frac{k_{z}}{k} \frac{d E}{d k} q_{z}+\frac{1}{2}\left[\frac{1}{k} \frac{d E}{d k}+\left(\frac{d^{2} E}{d k^{2}}-\frac{1}{k} \frac{d E}{d k}\right)\right. \\
& \left.\times\left(\frac{k_{z}}{k}\right)^{2}\right] q_{z}^{2} .
\end{aligned}
$$

The coefficients $G(k)$ and $H(k)$ in Eq. (19) are expressed in terms of $a_{\mathbf{k}}, b_{\mathbf{k}}$, and $c_{\mathbf{k}}$. We do not need their explicit expressions in this calculation. We should note that the $q_{z}$-linear term vanishes in the expansion of $I_{\mathbf{k}, \mathbf{k} \pm \mathbf{q}}$. Substi- 
TABLE I. Parameter values of InSb used in the present calculation.

\begin{tabular}{lll}
\hline \hline$E_{G}$ & \multicolumn{1}{c}{$0.1677 \mathrm{eV}$ (Ref. 20) } & $\begin{array}{l}\text { Band-gap energy at } T=300 \mathrm{~K} \\
\text { Spin-orbit splitting }\end{array}$ \\
$\Delta_{S O}$ & $0.850 \mathrm{eV}$ (Ref. 21) & $\begin{array}{c}\text { Band-edge effective mass in units } \\
\text { of the free-electron mass }\end{array}$ \\
$m_{0}^{*} / m_{0}$ & 0.01359 (Refs. 22,23) & High-frequency dielectric constant \\
& 15.68 (Ref. 24) & Static dielectric constant \\
$\varepsilon_{\infty}$ & 17.88 (Ref. 24) & Transverse optical-phonon energy \\
$\varepsilon_{0}$ & $22.90 \mathrm{meV}$ (Ref. 24) & Phonon relaxation-rate constant \\
$\hbar \omega_{T O}$ & $0.007 \omega_{T O}(0.16 \mathrm{meV})($ Ref. 24) & Relaxation-rate constant for \\
$\gamma(\hbar \gamma)$ & $3 \times 10^{12} \mathrm{~s}^{-1}(1.97 \mathrm{meV})$ & electronic transitions \\
$\eta(\hbar \eta)$ & & \\
\hline \hline
\end{tabular}

tuting Eqs. (19) and (20) into Eq. (18) and performing angular integration, we can obtain the following simple expression of $\varepsilon(\mathbf{q}=0, \omega)$ :

$$
\varepsilon(\mathbf{q}=0, \omega)=\varepsilon_{\infty}+\frac{\left(\varepsilon_{0}-\varepsilon_{\infty}\right) \omega_{T O}^{2}}{\omega_{T O}^{2}-\omega^{2}}-\frac{4 \pi n_{0} e^{2}}{m^{*} \omega^{2}},
$$

where the effective mass $m^{*}$ is defined by

$$
\frac{1}{m^{*}}=\frac{\beta}{3 \pi^{2} \hbar^{2} n_{0}} \int_{0}^{\infty} d k k^{2} f(k)[1-f(k)]\left[\frac{d E(k)}{d k}\right]^{2} .
$$

In Eq. (22) we employ the energy dispersion $E(k)$ obtained from Eq. (2). The symbol $\beta$ is defined by $\beta=1 / k_{B} T$ in terms of the Boltzmann constant $k_{B}$ and the absolute temperature $T$. We should note that the effective mass $m^{*}$ in Eq. (22) is determined from the energy dispersion around the Fermi level $\mu$, because the factor $f(k)[1-f(k)]$ becomes appreciable around a wave number $k$ corresponding to $\mu$. The mode energies at $q=0$ can be obtained from $\varepsilon(\mathbf{q}=0, \omega)$ $=0$, and the $\omega$ dependence of $\varepsilon(\mathbf{q}=0, \omega)$ is related to the infrared-reflection spectrum.

The nonparabolicity of the conduction band is treated in a complete manner in the above-mentioned scheme. As stated in Sec. I, we call this scheme the CNP one. We compare the results of this scheme with those of two simplified schemes. In one of these two schemes, we neglect the spin-orbit splitting of the valence band. ${ }^{12,19}$ The conduction-band dispersion $E(\mathbf{k})$ is given by the larger solution of an algebraic equation for $E$ :

$$
E(1+\alpha E)=\hbar^{2} \mathbf{k}^{2} / 2 m_{0}^{*},
$$

where $\alpha$ is defined by $\alpha=1 / E_{G}$. The overlap integral $I_{\mathbf{k}, \mathbf{k}^{\prime}}$ is expressed as

$$
I_{\mathbf{k}, \mathbf{k}^{\prime}}=\left(a_{\mathbf{k}} a_{\mathbf{k}^{\prime}}+c_{\mathbf{k}} c_{\mathbf{k}^{\prime}} \cos \theta_{\mathbf{k}, \mathbf{k}^{\prime}}\right)^{2}
$$

in terms of

$$
a_{\mathbf{k}}=\left[\frac{1+\alpha E(\mathbf{k})}{1+2 \alpha E(\mathbf{k})}\right]^{1 / 2}
$$

and

$$
c_{\mathbf{k}}=\left[\frac{\alpha E(\mathbf{k})}{1+2 \alpha E(\mathbf{k})}\right]^{1 / 2} .
$$

This is the SNP scheme, as named in Sec. I. In the other of the two simplified schemes, we assume a parabolic dispersion of the conduction band with the effective mass $m^{*}$ in Eq. (22). The parabolic dispersion with $m *$ leads to the same expression of $\varepsilon(\mathbf{q}=0, \omega)$ as in Eq. (21). Accordingly, even the parabolic scheme can give the same energy values of excitation modes at $q=0$ as the CNP scheme, if we employ the effective mass $m^{*}$ defined by Eq. (22) with $E(\mathbf{k})$ obtained by the CNP scheme. The parabolic dispersion $E(\mathbf{k})$ $=\hbar^{2} \mathbf{k}^{2} / 2 m^{*}$ is substituted into Eq. (12), and the overlap integral $I_{\mathbf{k}, \mathbf{k} \pm \mathbf{q}}$ is set to unity. We refer to this parabolic scheme with the effective mass modified as the MP one.

\section{RESULTS AND DISCUSSION}

By means of the theoretical framework in Sec. II, we investigate those electronic excitations in the nonparabolic conduction band which are coupled with polar phonons. As an example for calculation, we adopt $n$-type InSb whose conduction band is highly nonparabolic. In the following calculations, we use parameter values tabulated in Table $\mathrm{I}^{20-24} \mathrm{We}$ take room temperature $T=300 \mathrm{~K}$. The band gap $E_{G}$ depends upon $T$ significantly, and the $E_{G}$ value used is for $T$ $=300 \mathrm{~K} .{ }^{20}$ By virtue of the Lyddane-Sachs-Teller relation, we obtain the longitudinal optical-phonon energy of long wavelength as $\hbar \omega_{L O}=\sqrt{\varepsilon_{0} / \varepsilon_{\infty}} \hbar \omega_{T O}=24.45 \mathrm{meV}$. In our calculations, we employ a small and specific value of $\eta$, namely, $\eta=3 \times 10^{12} \mathrm{~s}^{-1} \quad(\hbar \eta=1.97 \mathrm{meV})$. As shown in Table I of Ref. 25, the relaxation time $\tau$ of carriers is estimated to be remarkably long in $n$-type InSb even at room temperature. As the carrier concentration $n_{0}$ increases from $3.5 \times 10^{17} \mathrm{~cm}^{-3}$ to $4.0 \times 10^{18} \mathrm{~cm}^{-3}$, the estimated value of $\tau^{-1}$ varies from $2.1 \times 10^{12} \mathrm{~s}^{-1}$ to $3.8 \times 10^{12} \mathrm{~s}^{-1}$. The above value of $\eta$ used in our calculations is located in the middle of this $\tau^{-1}$ range. The conspicuously long relaxation time is responsible for the fact that the infrared-reflection spectrum observed exhibits a sharp minimum with negligible intensity. $^{25}$

Figure 1 shows the energy dispersion of the conduction band in the CNP scheme (full curve), that in the SNP scheme (dash-dotted curve), that in the MP scheme (broken curve), and the parabolic $(P)$ dispersion with the band-edge effective mass $m_{0}^{*}$ (dotted curve). The CNP curve and the SNP one are obtained from Eqs. (2) and (23), respectively. With an increase in $k$, the CNP curve and the SNP one start to deviate 


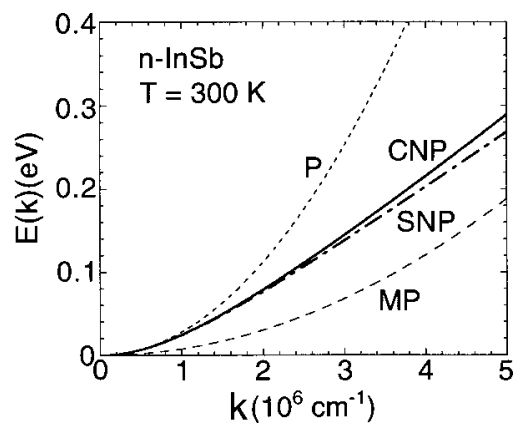

FIG. 1. Energy dispersion of the conduction band of InSb at $T$ $=300 \mathrm{~K}$ obtained by the complete nonparabolic (CNP) scheme (full curve), the simplified nonparabolic (SNP) one (dash-dotted curve), and the modified parabolic (MP) one (broken curve), in comparison with the parabolic $(P)$ dispersion with the band-edge effective mass (dotted curve). The dispersion curve in the MP scheme is for carrier concentration $n_{0}=4.0 \times 10^{18} \mathrm{~cm}^{-3}$.

from the $P$ curve remarkably, and evolve into a linearlike increase. This remarkable deviation indicates a high nonparabolicity. The SNP curve lies somewhat below the CNP one. Although this departure looks small, in the below analysis, we will find a considerable difference in excitations between these two nonparabolic schemes. For the MP curve, we employ the modified effective mass $m^{*}$ that is obtained from Eq. (22) at carrier concentration $n_{0}=4.0 \times 10^{18} \mathrm{~cm}^{-3}$. Because of the high nonparabolicity, the MP curve is unrealistically below the CNP curve. However, as seen from the below-mentioned analysis, the important point is that the $k$ derivative of the MP curve around its Fermi level $\mu$ $=179 \mathrm{meV}$ is almost the same as that of the CNP curve around $\mu=278 \mathrm{meV}$.

Here, following Ref. 12, we pay attention to an intensityvanishing point in the infrared-reflection spectrum (a deep minimum with negligible intensity). The $\omega$ dependence of $\varepsilon(\mathbf{q}=0, \omega)$ is related to this spectrum, and energy values $\hbar \omega$ satisfying $\varepsilon(\mathbf{q}=0, \omega)=1$ correspond to the intensityvanishing points in the spectrum. The dielectric function $\varepsilon(\mathbf{q}=0, \omega)$ is given by Eq. (21). Figure 2 exhibits the $n_{0}$ dependence of the higher energy $\hbar \omega$ of $\varepsilon(\mathbf{q}=0, \omega)=1$ in the CNP scheme (full curve), the SNP scheme (dash-dotted curve), and the parabolic scheme with the band-edge effective mass $m_{0}^{*}$ (dotted curve), in comparison with the experimental result (five dots). ${ }^{25}$ In the CNP (SNP) scheme, the effective mass $m^{*}$ in Eq. (22) is calculated by using $E(k)$ in the CNP (SNP) scheme, and in the parabolic scheme, $m^{*}$ in

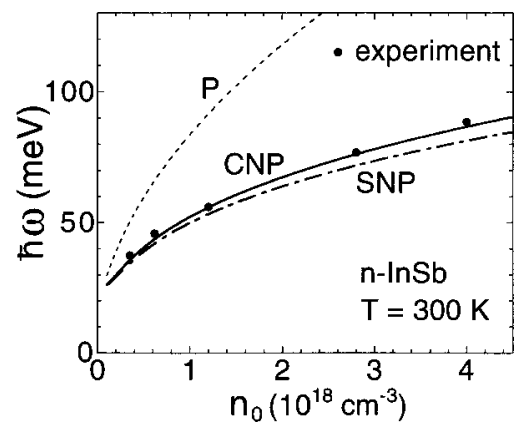

FIG. 2. Carrier-concentration dependence of the higher one of the two energy values satisfying $\varepsilon(\mathbf{q}=0, \omega)=1$ for $n$-type InSb at $T=300 \mathrm{~K}$. The calculated results in the CNP scheme (full curve), the SNP one (dash-dotted curve), and the parabolic one with the band-edge effective mass (dotted curve) are compared with intensity-vanishing points in observed infrared-reflection spectra (five dots).

Eq. (21) is replaced by $m_{0}^{*}$. In this analysis, the MP scheme is equivalent to the CNP one. As displayed in Fig. 2, the energy values in the CNP scheme are in excellent agreement with the experimental ones, and the energy values in the SNP scheme are appreciably lower than those in the CNP scheme in a higher $n_{0}$ region. Equation (22) implies that $m^{*}$ is determined by the $k$ derivative of $E$ around the Fermi level $\mu$. Table II compiles the $\mu$ and $m^{*}$ values in the CNP, SNP, and MP schemes for four carrier concentrations. The Fermi level for each $n_{0}$ value can be located in the conduction-band dispersion of Fig. 1. At higher carrier concentrations, the $k$ derivative around $\mu$ becomes appreciably lower in the SNP scheme than in the CNP scheme, and consequently the effective mass $m^{*}$ in the SNP scheme becomes larger than that in the CNP scheme. This explains why the energy at the intensity-vanishing point in the SNP scheme is appreciably lower than that in the CNP scheme in a higher $n_{0}$ range.

We can obtain the energy dispersion of each excitation mode by locating the corresponding peak in $\omega$ dependence of $F$ at various $q$ values. Figure 3 displays the energy-dispersion diagrams of excitation modes at $n_{0}=4.0 \times 10^{18} \mathrm{~cm}^{-3}$ (a), $1.0 \times 10^{18} \mathrm{~cm}^{-3}$ (b), $0.5 \times 10^{18} \mathrm{~cm}^{-3}$ (c), and 0.1 $\times 10^{18} \mathrm{~cm}^{-3}$ (d). Full curves, dash-dotted ones, and broken ones correspond to the CNP scheme, the SNP one, and the MP one, respectively. At each $n_{0}$ value, there exist three dispersion branches, which we name $A, B$, and $C$. In (a), (b), or (c), the curves of the branch $B$ in the three schemes coincide with one another, and look like one curve. In each panel, the curves of the branch $C$ in the three schemes also agree

TABLE II. Carrier-concentration dependence of the Fermi level $\mu$ measured from the conduction-band bottom and the effective mass $m^{*}$ defined by Eq. (22) in the CNP, SNP, and MP schemes.

\begin{tabular}{lrrrr}
\hline \hline$n_{0}\left(10^{18} \mathrm{~cm}^{-3}\right)$ & 0.1 & 0.5 & 1.0 & 4.0 \\
$\mu(\mathrm{meV})$ in CNP & 23.2 & 96.2 & 142.1 & 277.7 \\
$\mu(\mathrm{meV})$ in SNP & 20.9 & 90.9 & 133.8 & 256.6 \\
$\mu(\mathrm{meV})$ in MP & 9.6 & 65.8 & 96.4 & 178.8 \\
$m^{*} / m_{0}$ in CNP & 0.0248 & 0.0308 & 0.0356 & 0.0505 \\
$m^{*} / m_{0}$ in SNP & 0.0266 & 0.0335 & 0.0391 & 0.0574 \\
$m^{*} / m_{0}$ in MP & 0.0248 & 0.0308 & 0.0356 & 0.0505 \\
\hline \hline
\end{tabular}



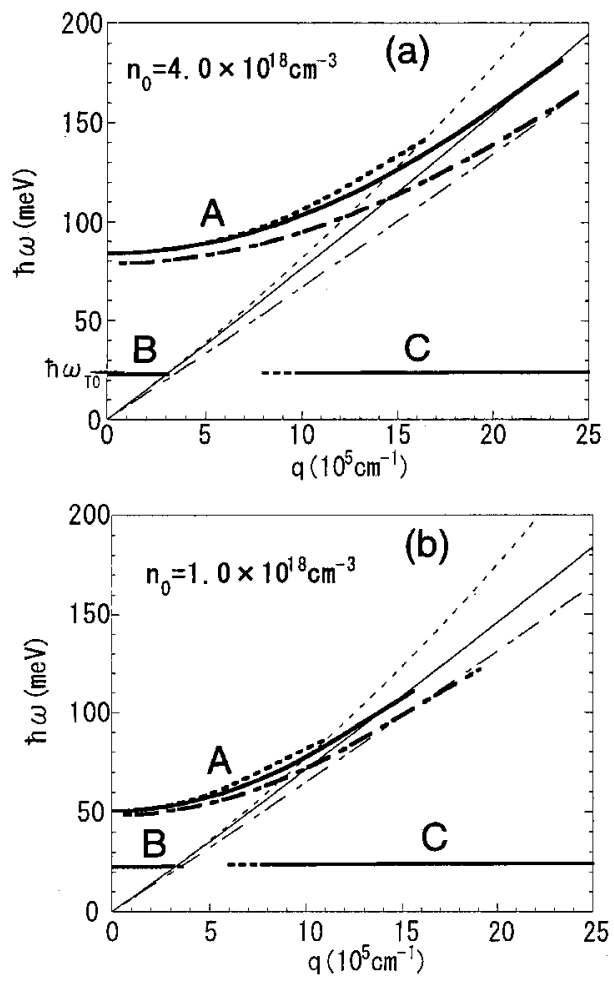
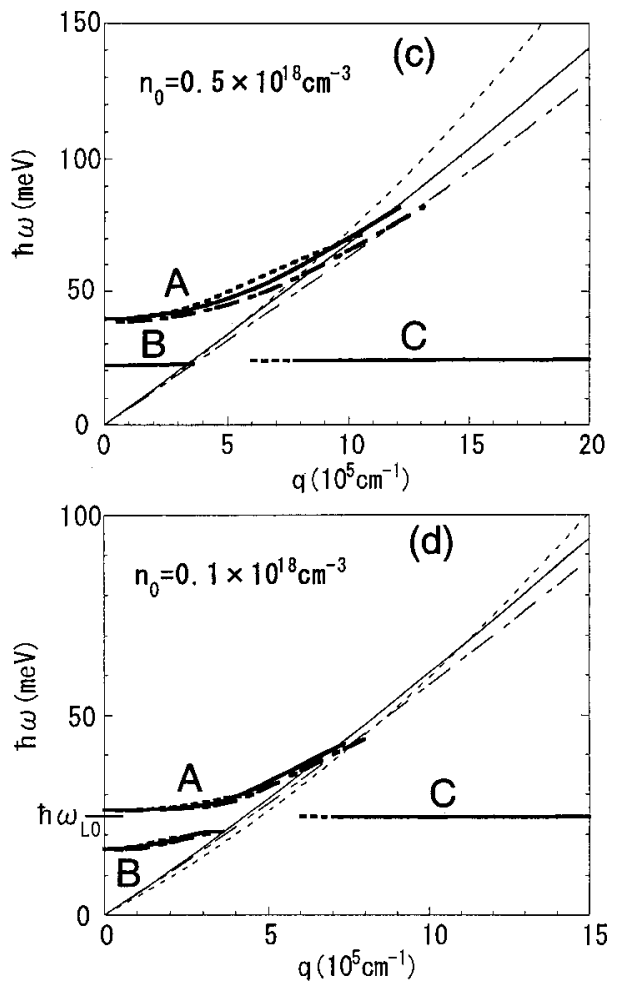

FIG. 3. Energy-dispersion diagrams of excitation modes of $n$-type $\mathrm{InSb}$ at $T=300 \mathrm{~K}$ for carrier concentration $n_{0}=4.0$ $\times 10^{18} \mathrm{~cm}^{-3}$ (a), $1.0 \times 10^{18} \mathrm{~cm}^{-3}$ (b), $0.5 \times 10^{18} \mathrm{~cm}^{-3}$ (c), and 0.1 $\times 10^{18} \mathrm{~cm}^{-3}$ (d). Full curves, dash-dotted ones, and broken ones correspond to the CNP, SNP, and MP schemes, respectively. At each $n_{0}$ value, there exist three dispersion branches $A, B$, and $C$. Each of the three curves (full, dash-dotted, and broken) starting from the origin marks the boundary of the single-particle excitation continuum in the corresponding scheme at $T=0 \mathrm{~K}$. The resonance intensity decays away on a broken part of the branch $C$. with one another. At $T=0 \mathrm{~K}$, the SPE continuum in each scheme has a definite boundary, and it extends on the right side of the corresponding one of the three curves starting from the origin. This boundary curve for $T=0 \mathrm{~K}$ helps us to realize around where the Landau damping begins to operate, though we calculate excitation modes at $T=300 \mathrm{~K}$, and the boundary becomes blurred to a certain extent then. At a higher $n_{0}$ value, the boundary curve in the SNP (MP) scheme begins to deviate downward (upward) conspicuously from that in the CNP scheme with increase in $q$. This deviation originates from the difference in the conduction-band dispersion among the three schemes (see Fig. 1). Figures 4, 5, and 6 show the $\omega$ dependence of $F$ at each of various $q$ values for the branch $A$ with $n_{0}=4.0 \times 10^{18} \mathrm{~cm}^{-3}$ [see Fig. 3(a)], the branch $B$ (and $A$ ) with $n_{0}=0.1 \times 10^{18} \mathrm{~cm}^{-3}$ [see Fig. 3(d)], and the branch $C$ with $n_{0}=1.0 \times 10^{18} \mathrm{~cm}^{-3}$ [see Fig. 3(b)], respectively. An $\omega$ range at a $q$ value specified in each panel can be located in Fig. 3. As in Fig. 3, full curves, dash-dotted ones, and broken ones correspond to the CNP, SNP, and MP schemes, respectively.

The branch $A$ is the upper one of the coupled plasmon (PL)-phonon (PH) modes. As the branch descends toward $\hbar \omega_{L O}(=24.45 \mathrm{meV})$ with decrease in $n_{0}$, the PL-like modes evolve into the PH-like ones. On the other hand, the branch $B$ is the lower one of the coupled PL-PH modes. As the branch falls further below $\hbar \omega_{T O}$ with decrease in $n_{0}$, the coupled modes involving strong cancellation in amplitude between the PL and PH components in the induced-charge density change into the PL-like modes. The remarkable variation in the mode structure with change in $n_{0}$ is examined closely in Ref. 10.

Here, we examine the difference in the dispersion of the branch $A$ or $B$ among the three schemes. In the branch $A$, we
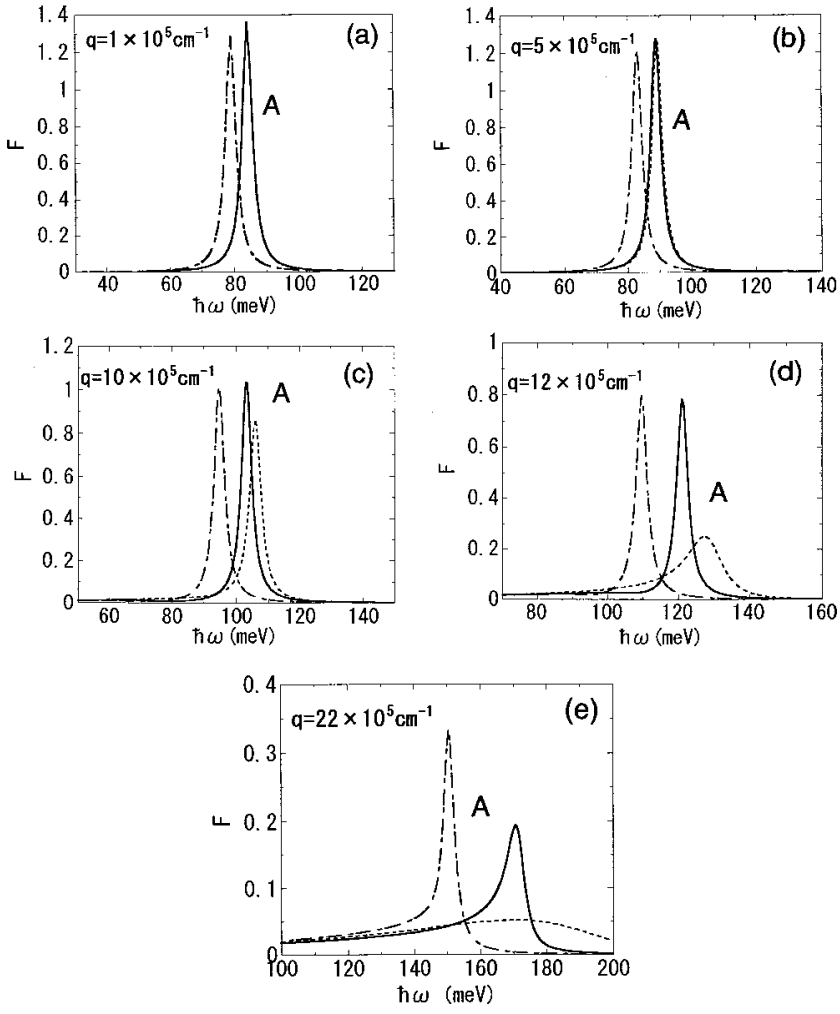

FIG. 4. $\omega$ dependence of the energy-loss function $F$ at $q=1$ $\times 10^{5} \mathrm{~cm}^{-1} \quad$ (a), $5 \times 10^{5} \mathrm{~cm}^{-1} \quad$ (b), $10 \times 10^{5} \mathrm{~cm}^{-1} \quad$ (c), 12 $\times 10^{5} \mathrm{~cm}^{-1}$ (d), and $22 \times 10^{5} \mathrm{~cm}^{-1}$ (e) for the branch $A$ of $n$-type $\mathrm{InSb}$ at $n_{0}=4.0 \times 10^{18} \mathrm{~cm}^{-3}$ and $T=300 \mathrm{~K}$. Full curves, dashdotted ones, and broken ones correspond to the CNP, SNP, and MP schemes, respectively. 

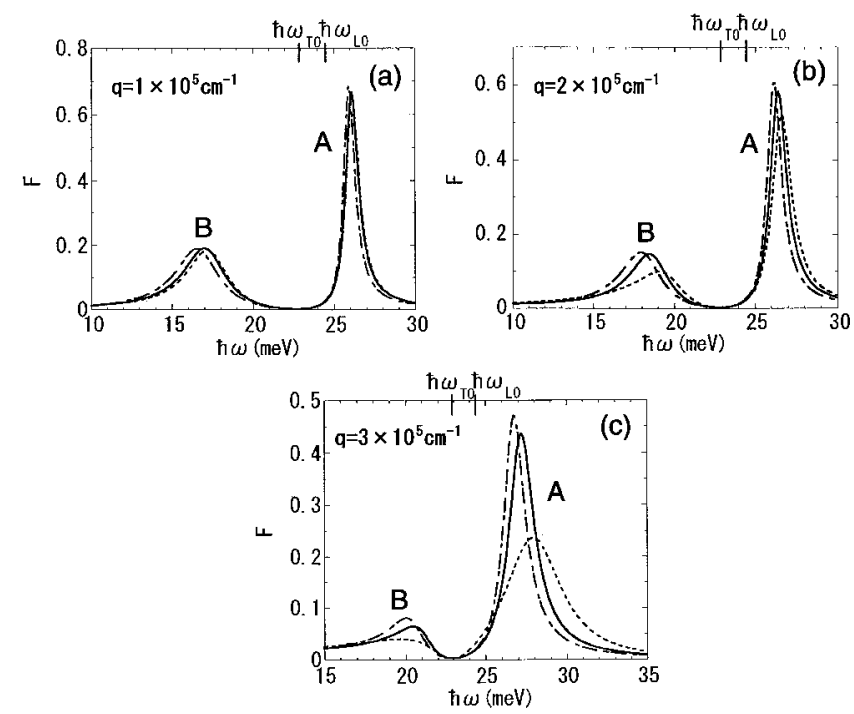

FIG. 5. $\omega$ dependence of $F$ at $q=1 \times 10^{5} \mathrm{~cm}^{-1}$ (a), 2 $\times 10^{5} \mathrm{~cm}^{-1}(\mathrm{~b})$, and $3 \times 10^{5} \mathrm{~cm}^{-1}$ (c) for the branch $B$ (and $A$ ) of $n$-type InSb at $n_{0}=0.1 \times 10^{18} \mathrm{~cm}^{-3}$ and $T=300 \mathrm{~K}$. Organized in the same manner as Fig. 4.

find a greater difference among the three schemes at a higher $n_{0}$ value, while, in the branch $B$, we notice a perceivable difference only at the lowest $n_{0}$ value. We pay attention to the branch $A$ at $n_{0}=4.0 \times 10^{18} \mathrm{~cm}^{-3}$ in Fig. 3(a) where the difference among the three schemes is most obvious. The branch in the SNP scheme (dash-dotted curve) lies considerably below that in the CNP scheme (full curve) over the whole $q$ range. With an increase in $q$, this deviation becomes larger, and the mode in the SNP scheme approaches the boundary of the SPE continuum at larger $q$ values than the mode in the CNP scheme. In Fig. 4, we can follow the variation in the resonance peak of the mode on the branch $A$ at the same $n_{0}$ value with change in $q$. When $q \leqslant 12 \times 10^{5} \mathrm{~cm}^{-1}$, there is no substantial difference in height and width of the resonance peak between the CNP and SNP schemes [see Figs. 4(a)-4(d)]. At $q=22 \times 10^{5} \mathrm{~cm}^{-1}$, however, the resonance peak in the CNP scheme becomes appreciably broad, though that in the SNP scheme shows no substantial broadening. This implies that the Landau damping begins to operate at smaller $q$ values in the CNP scheme than in the SNP scheme. This is consistent with the above-mentioned approach to the boundary of the SPE continuum at different $q$ values. In passing, an extending tail on the lower-energy side of each resonance peak in Fig. 4(e) arises from the SPEs.

Next, we analyze the difference in the branch $A$ between the CNP and MP schemes. As exhibited in Fig. 3(a), with an increase in $q$, the branch in the MP scheme starts to deviate upward from that in the CNP scheme, and this deviation becomes larger. The mode in the MP scheme reaches the boundary of the SPE continuum at smaller $q$ values than the mode in the CNP scheme. When $q \leqslant 5 \times 10^{5} \mathrm{~cm}^{-1}$, the resonance peak in the MP scheme accords completely or almost completely with that in the CNP scheme [see Figs. 4(a) and 4(b)]. With further increase in $q$, however, the former peak departs from the latter peak to the higher-energy side, and begins to broaden at smaller $q$ values than the latter peak. At
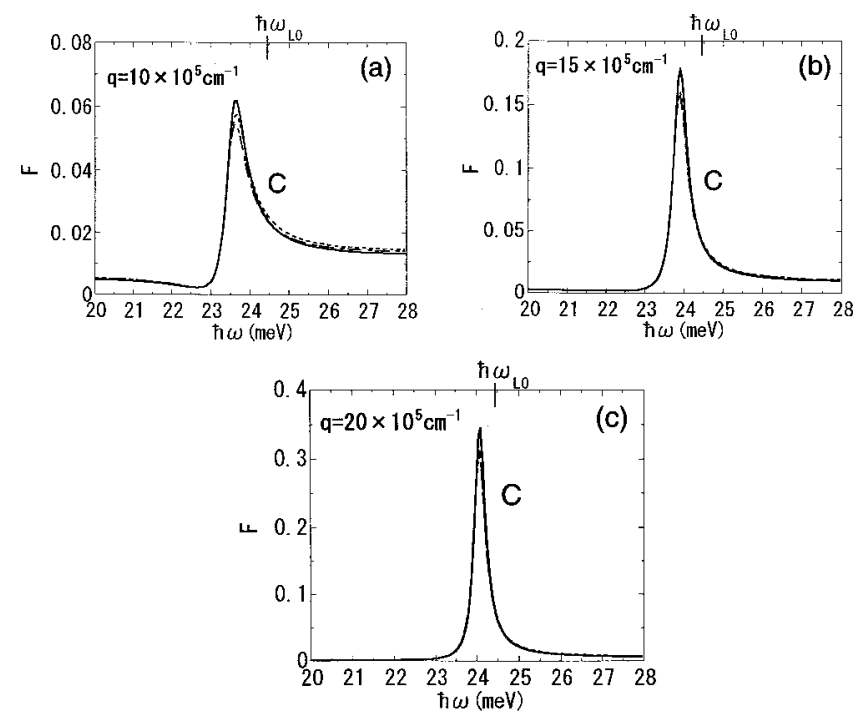

FIG. 6. $\omega$ dependence of $F$ at $q=10 \times 10^{5} \mathrm{~cm}^{-1}$ (a), 15 $\times 10^{5} \mathrm{~cm}^{-1}$ (b), and $20 \times 10^{5} \mathrm{~cm}^{-1}$ (c) for the branch $C$ of $n$-type InSb at $n_{0}=1.0 \times 10^{18} \mathrm{~cm}^{-3}$ and $T=300 \mathrm{~K}$. Laid out in the same fashion as Fig. 4.

$q=22 \times 10^{5} \mathrm{~cm}^{-1}$, the former peak decays away into the weak extending intensity due to the SPEs [see Fig. 4(e)]. This indicates that the Landau damping comes into play at considerably smaller $q$ values in the MP scheme than in the CNP scheme.

Here, we turn our attention to the energy dispersion and the energy-loss intensity of the branch $B$ with $n_{0}=0.1$ $\times 10^{18} \mathrm{~cm}^{-3}$ in Fig. 3(d) and Fig. 5, respectively. The difference among the three schemes in the branch $B$ is similar to that in the branch $A$, though the former is not so evident as the latter. As displayed in Fig. 3(d), the branch in the SNP scheme lies somewhat below that in the CNP scheme, and with increase in $q$, the branch in the MP scheme starts to show a small upward deviation from that in the CNP scheme. As exhibited in Fig. 5, the Landau damping in the SNP (MP) scheme begins to operate at larger (smaller) $q$ values than that in the CNP scheme. The Landau damping at smaller $q$ values in the MP scheme seems to be inconsistent with the fact that the mode on this branch gets close to the boundary of the SPE continuum at larger $q$ values in this scheme [see Fig. 3(d)]. However, we should note that the value of $\mu$ $(=9.6 \mathrm{meV})$ measured from the conduction-band bottom (see Table II) is considerably lower than $k_{B} T(=25.9 \mathrm{meV})$, and that the carrier system begins to acquire classical-gas character. This is considered to account for the fact that the Landau damping comes into play at smaller $q$ values in the MP scheme. Incidentally, in the branch $A$ at the same $n_{0}$ value as well, we can find a similar quick damping in the MP scheme (see Fig. 5).

Next, we investigate the branch $C$ that lies flat just below $\hbar \omega_{L O}(=24.45 \mathrm{meV})$. As shown in Fig. 3, at any of the four $n_{0}$ values, there is no difference in the energy dispersion among the three schemes. With decrease in $q$, the resonance intensity declines, and decays away on a broken part of the dispersion curve. Figure 6 exhibits the variation in the reso- 
nance peak at $n_{0}=1.0 \times 10^{18} \mathrm{~cm}^{-3}$ with change in $q$. In this branch, the longitudinal optical-phonon polarization plays a major role, and the carriers have a screening effect on the phonon polarization. The carriers operate against the phonon polarization to reduce the energy-loss intensity. ${ }^{10}$ As displayed in Fig. 6(a), at $q=10 \times 10^{5} \mathrm{~cm}^{-1}$, the reduced resonance intensity forms a declining peak in the extending intensity due to the SPEs. As the screening effect becomes less powerful with increase in $q$, the mode energy asymptotically approaches the value of $\hbar \omega_{L O}$, and the resonance intensity of $F$ increases toward that in the absence of carriers.

As shown in Fig. 1, the conduction-band dispersion has a somewhat smaller $k$ derivative around $\mu$ in the SNP scheme than in the CNP scheme. This smaller derivative operates to enhance the screening effect, and consequently, to suppress the energy-loss intensity. In the MP scheme, the overlap integral $I_{\mathbf{k}, \mathbf{k} \pm \mathbf{q}}$ is set to unity, though, in the CNP or SNP scheme, it can be less than unity in a larger $q$ region. No decrease in the overlap integral in the MP scheme acts to overestimate the screening effect, and as a consequence, to reduce the energy-loss intensity. These two effects of the energy dispersion and the overlap integral explain why the resonance intensity in the SNP or MP scheme is somewhat weaker than that in the CNP scheme.

Finally, we mention the validity of the RPA in our calculations. We can evaluate the exchange-correlation (XC) effect by means of the so-called local-field correction. ${ }^{26,27}$ The XC effect operates to weaken the upward PL dispersion without making any energy change at $q=0$. The XC effect has no influence on the mode energies at $q=0$ and the intensity-vanishing point in the infrared-reflection spectrum. The above effect of lowering the PL energy in a larger $q$ region depends upon the effective electron density significantly. Using $m^{*} / m_{0}$ in the CNP scheme in Table II and $\varepsilon_{0}$ in Table I, we can estimate the effective Bohr radius $a_{B}^{*}$ and the effective density parameter $r_{s}^{*}$ defined by $(4 \pi / 3)\left(r_{s}^{*} a_{B}^{*}\right)^{3} n_{0}=1$. The $r_{s}^{*}$ value varies from 0.21 to
0.35 , when $n_{0}$ decreases from $4.0 \times 10^{18} \mathrm{~cm}^{-3}$ to 0.1 $\times 10^{18} \mathrm{~cm}^{-3}$. Accordingly, the $r_{s}^{*}$ value remains considerably lower than unity in the whole $n_{0}$ range analyzed here. This indicates that the XC effect is quite small in our carrier system of high effective density.

\section{SUMMARY}

Incorporating a nonparabolic conduction-band dispersion into the random-phase approximation in a complete manner, we have examined those electronic excitations in the nonparabolic conduction band of an $n$-type narrow-gap semiconductor which are coupled with polar phonons. Our results of this complete nonparabolic (CNP) scheme have been compared with those of two simplified schemes, namely, a simplified nonparabolic (SNP) scheme with the spin-orbit splitting neglected and a modified parabolic (MP) scheme using a modified effective mass. Our analysis has shown that the complete treatment of the nonparabolicity as in the CNP scheme is essential to accurate evaluation of the energy dispersion, the energy-loss intensity, and the Landau damping of the plasmonlike mode at higher carrier concentrations. The dispersion branch of this mode in the SNP scheme lies considerably below that in the CNP scheme, and with increase in wave number $q$, the dispersion branch in the MP scheme begins to deviate upward from that in the CNP scheme. The Landau damping starts to operate at larger (smaller) $q$ values in the SNP (MP) scheme than in the CNP scheme. We have obtained an excellent agreement between the CNP scheme and the experiment on the carrierconcentration dependence of an intensity-vanishing point in the infrared-reflection spectrum.

\section{ACKNOWLEDGMENTS}

Part of the numerical calculations in the present work were performed at the Computer Center of Iwate University and the Information Synergy Center of Tohoku University.
*Present address: Aomori River Techno Co. Ltd., 245-11 Yamaguchi, Nogi, Aomori, Aomori 030-0142, Japan.

${ }^{\dagger}$ To whom correspondence should be addressed.

${ }^{1}$ W. Richter, in Polarons and Excitons in Polar Semiconductors and Ionic Cristals, edited by J. T. Devreese and F. Peeters (Plenum, New York, 1984), pp. 209-243, and references therein.

${ }^{2}$ G. Abstreiter, M. Cardona, and A. Pinczuk, in Light Scattering in Solids IV, edited by M. Cardona and G. Güntherodt (Springer, Berlin, 1984), pp. 5-150, and references therein.

${ }^{3}$ J. Lindhard, K. Dan. Vidensk. Selsk. Mat. Fys. Medd. 28, 8 (1954).

${ }^{4}$ L. F. Lemmens and J. T. Devreese, Solid State Commun. 14, 1339 (1974)

${ }^{5}$ S. Katayama, K. Murase, and H. Kawamura, Solid State Commun. 16, 945 (1975).

${ }^{6}$ L. F. Lemmens, F. Brosens, and J. T. Devreese, Solid State Commun. 17, 337 (1975).

${ }^{7}$ G. Abstreiter, R. Trommer, M. Cardona, and A. Pinczuk, Solid State Commun. 30, 703 (1979).

${ }^{8}$ T. Yuasa, S. Naritsuka, M. Mannoh, K. Shinozaki, K. Yamanaka,
Y. Nomura, M. Mihara, and M. Ishii, Phys. Rev. B 33, 1222 (1986).

${ }^{9}$ W. Richter, U. Nowak, H. Jürgensen, and U. Rössler, Solid State Commun. 67, 199 (1988).

${ }^{10}$ T. Inaoka, J. Phys.: Condens. Matter 3, 4825 (1991).

${ }^{11}$ S. Ernst, A. R. Goñi, K. Syassen, and M. Cardona, Phys. Rev. B 53, 1287 (1996).

${ }^{12}$ M. P. Hasselbeck and P. M. Enders, Phys. Rev. B 57, 9674 (1998).

${ }^{13}$ L. Artús, R. Cuscó, J. Ibáñez, N. Blanco, and G. González-Díaz, Phys. Rev. B 60, 5456 (1999).

${ }^{14}$ E. O. Kane, J. Phys. Chem. Solids 1, 249 (1957).

${ }^{15}$ U. Rössler, Solid State Commun. 49, 943 (1984).

${ }^{16}$ T. Inaoka, Surf. Sci. 431, 156 (1999).

${ }^{17}$ M. Kim, A. Das, and S. Senturia, Phys. Rev. B 18, 6890 (1978).

${ }^{18}$ D. Matz, Phys. Rev. 168, 843 (1968).

${ }^{19}$ W. Fawcett, A. D. Boardman, and S. Swain, J. Phys. Chem. Solids 31, 1963 (1970).

${ }^{20}$ C. L. Littler and D. G. Seiler, Appl. Phys. Lett. 46, 986 (1985). 
${ }^{21}$ S. Logothetidis, L. Vina, and M. Cardona, Phys. Rev. B 31, 947 (1985).

${ }^{22}$ D. M. Zengin, J. Phys. D 16, 653 (1983).

${ }^{23}$ R. A. Isaacson, Phys. Rev. 169, 312 (1968).

${ }^{24}$ M. Hass and B. W. Henvis, J. Phys. Chem. Solids 23, 1099
(1962).

${ }^{25}$ W. G. Spitzer and H. Y. Fan, Phys. Rev. 106, 882 (1957).

${ }^{26}$ K. S. Singwi, M. P. Tosi, R. H. Land, and A. Sjölander, Phys. Rev. 176, 589 (1968).

${ }^{27}$ A. Gold and L. Calmels, Phys. Rev. B 48, 11622 (1993). 